\title{
APPLICATION OF IMMUNOHISTOGHEMISTRY FOR DIAGNOSIS OF NEOPLASMS: MUCIN ANTIGENS EXPRESSION AND BIOLOGICAL BEHAVIOR OF NEOPLASMS
}

\author{
SUguru YONEZAWA \\ Second Department of Pathology, Kagoshima University School of Medicine, Kagoshima 890
}

\begin{abstract}
Mucins are high molecular weight glycoproteins having oligosaccharides attached to serine or threonine residues of the core protein backbone. Synthesis and secretion of mucin are common features of glandular epithelial tissues. Alterations in the glycosylation of mucins have been described in cancer, however little is known about the expression of different types of mucin core protein. To evaluate whether mucin antigens expression is correlated with the biological behavior of neoplasms or not, we examined the expression of mucin carbohydrate antigens associated with the earliest steps in mucin glycosylation (Tn and sialosyl-Tn) and core protein antigens associated with MUC1 and MUC2 gene products (DF3 and MRP) in tumors of several organs using immunohistochemical methods. Simultaneous expression of $\mathbf{T n}$ and sialosyl-Tn was observed in adenocarcinomas of the pancreas, intrahepatic bile-duct and ovary, in $100 \%$, $92 \%$ and $100 \%$ of the cases respectively. It was not frequently observed in the breast cancers $(10 \%)$, although a close association of $T$ n expression with lymph node metastasis was observed in the scirrhous type of breast cancer. In the pancreatic and intrahepatic bile-duct carcinomas, a pattern of DF3( +$)$ and MRP(-) was found in $100 \%$ of the invasive carcinomas with a poor prognosis, whereas DF3(-) and MRP $(+)$ pattern was seen in $79 \%$ of the non-invasive carcinomas with a favorable prognosis. In the breast cancers, DF 3 was expressed in $95 \%$ but MRP was not expressed. In the ovary, MRP was expressed only in the mucinous tumors, but not in the serous tumors. Ovarian serous adenocarcinomas showed DF3( +$)$ and MRP $(-)$ pattern in $100 \%$. On the other hand, ovarian mucinous adenocarcinomas showed DF3 $(+)$ and MRP (-) pattern in $45 \%$ of the cases, and DF3 $(+)$ and MRP $(+)$ pattern in $55 \%$, while benign mucinous adenomas did not show DF3(+) and MRP(+) pattern. These results suggest that the patterns of expression of several mucin antigens are associated with the biological properties of neoplasms, and are useful to predict prognosis of the patients.
\end{abstract}

Mucins are high molecular weight glycoproteins having oligosaccharides attached to serine or threonine residues of the apomucin protein backbone by $\mathrm{O}$ glycosidic linkages (2). Synthesis and secretion of mucin is a common feature of glandular epithelial tissues, and alterations in the glycosylation of mucins have been described in cancer $(2,4,7,11)$. These alterations include aberrant glycosylation resulting in disaccharides such as sialosyl-Tn (STn) antigen $(2,4)$ and incomplete glycosylation resulting in accumulation of core oligosaccharide structures such as Tn antigen $(2,7)$.

Recently, it has been reported that many of the

Presented in part at the symposium 3 on Application of Histochemistry in Pathological Diagnosis, July 15, 1994 as part of the program at the 4th Joint Meeting of the Japan Society of Histochemistry and Cytochemistry and the Histochemical Society, held in Maui, Hawaii, July 13 to 16, 1994. cancer-associated antigens are mucin antigens $(2,3)$. We also reported that STn antigen was expressed frequently in many kinds of adenocarcinomas (11). Besides the extensive studies on carbohydrate chains of mucin antigens, the biochemical studies on the structures and the organ specificities of mucin core proteins have been reported recently $(1,3,10)$. However, there have been no systemic reports describing the expression of carbohydrate chains and core proteins of mucin antigens in various human cancers. We are interested in investigating the relationship between the mucin antigens expression in various human cancers and the biological behaviors of the cancers. This article is a review of our investigations on the expression of the carbohydrate antigens which are associated with the earliest steps in mucin glycosylation (Tn and STn) and the expression of the mucin core protein antigens associated with MUC1 gene product (mammary-type apomucin) and MUC2 gene product (intestinal-type 
apomucin) in neoplasms of pancreas (6), intrahepatic bile duct (9), ovary (8), and breast (5).

\section{MATERIALS AND METHODS}

Lists of neoplasms examined in our studies were described in each section I-IV. Table 1 is a list of antibodies that we used. Immunohistochemical staining was done on formalin-fixed paraffin-embedded tissue sections by an immunoperoxidase method using ABC procedure as described elsewhere $(5,6,8,9)$. The results of the antibodies stainings were graded by the percentage of positively stained neoplastic cells as follows: - , less than $5 \%$ of neoplastic cells stained; ,$+ 5-50 \%$ of neoplastic cells stained; and $H$, over $50 \%$ of the neoplastic cells stained. We evaluated the stainings of cytoplasm, cell apex (luminal surface), and associated secretory products (luminal contents) independently. Cells were considered positive when at least one of these components was positive.

\section{RESULTS}

\section{Pancreas}

At first, pancreatic tumors (intraductal papillary tumor 5, invasive ductal carcinoma 36) were studied (6), since MUC1 gene expression of human pancreatic cancer cell lines was reported in our previous study (10). In human pancreas, besides common invasive ductal carcinomas, intraductal papillary tumors were occasionally found. The intraductal papillary tumor reveals dilatation of the pancreatic duct, swelling of the papilla of Vater, and conspicuous mucus secretion, and usually shows expansive growth but rarely invasive growth. Fig. 1 shows staining results in each patient, with follow-up data, lymphatic permeation status in and around the pancreas, and lymph node metastasis status. There was a distinct difference between the survival of the patients with intraductal papillary tumor and that with invasive ductal carcinoma. Tn and STn were completely negative in the normal pancreatic tissue, whereas both antigens were always expressed in both invasive ductal carcinoma and intraductal papillary tumor. There was a clear contrast of the immunohistochemical stainings of MUC1 gene product (DF3) and MUC2 gene product (MRP) between invasive ductal carcinoma and intraductal papillary tumor. Invasive ductal carcinoma was DF3 positive but MRP negative, whereas intraductal papillary tumor was DF3 negative but MRP positive (Fig. 2). Thus, the MRP expression was seen in the tumors with good outcome and less aggressive lymphatic status, whereas the DF3 expression was seen in the tumors with poor outcome and aggressive

TABLE 1. Antibodies used to detect antigens

\begin{tabular}{ll}
\hline Antigen & Reagent used to detect antigen \\
\hline Tn(GalNAc-R) & 91S8(MAb) \\
Sialosyl-Tn(SA $\alpha-2,6$ GalNAc-R) & TKH2(MAb) \\
MUC1-apomucin-related antigen & DF3(MAb), MUSE11(MAb)*, 139H2(MAb)* \\
MUC2-apomucin-related antigen & Anti-MRP(PAb), CCP58(MAb)* \\
\hline
\end{tabular}

GalNAc: N-acetylgalactosamine, SA: sialic acid,

MAb: monoclonal antibody, PAb: polyclonal antibody

* used only for the study of intrahepatic bile-duct tumors

91S8: mouse IgM, ascites, provided by Dr. Y. S. Kim, Veterans Administration Medical Center, San Francisco, CA, U.S.A.

TKH2: mouse IgG, hybridoma culture supernatant, provided by Dr. Sen-itiroh Hakomori, The Biomembrane Institute, Seattle, WA, U.S.A.

DF3: mouse IgG, ascites, Centocor CA15-3, Toray-Fuji Bionics, Tokyo, Japan

MUSE11: mouse IgM, hybridoma culture supernatant, provided by Dr. Kohzoh Imai, Sapporo Medical College, Sapporo, Japan

139H2: mouse IgG, ascites, provided by Dr. John Hilkens, The Netherlands Cancer Institute, Amsterdam, The Netherlands

Anti-MRP: purified rabbit IgG, provided by Dr. Y. S. Kim

CCP58: mouse IgG, ascites, provided by Dr. Ian F. G. McKenzie, Austin Research Institute, Heidelberg, Victoria, Australia 

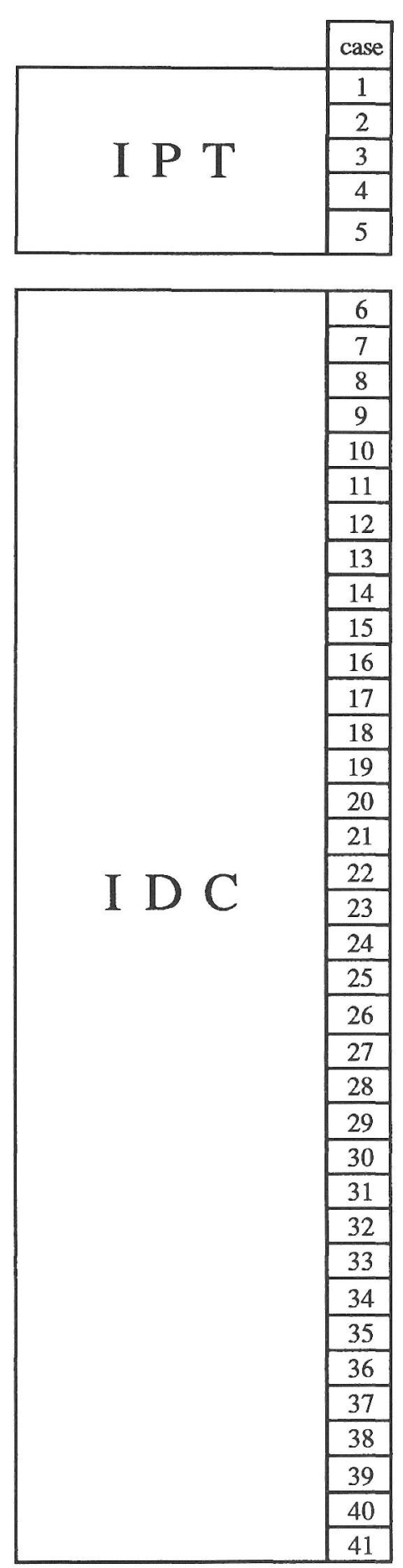

IPT : Intraductal papillary tumor IDC : Invasive ductal carcinoma

\begin{tabular}{|c||c||c|}
\hline MUC1 & MUC2 \\
\hline DF3 & anti-MRP \\
\hline 9158 & TKH2 \\
\hline
\end{tabular}
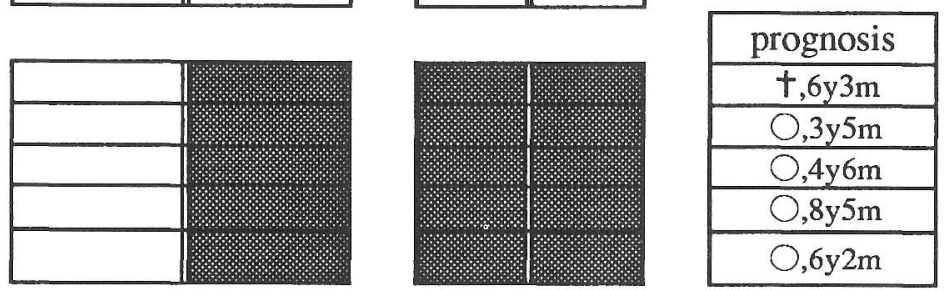

\begin{tabular}{|c|c|}
\hline ly & $\mathrm{n}$ \\
\hline 0 & 0 \\
\hline 0 & 0 \\
\hline 0 & 0 \\
\hline 1 & 0 \\
\hline 1 & 1 \\
\hline
\end{tabular}
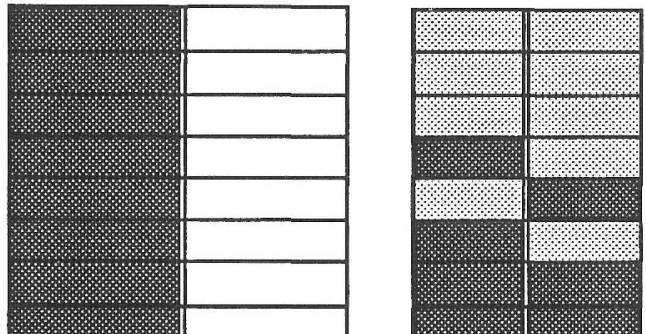

$+, 1$

$+, 1.5 \mathrm{~m}$

$+, 9 \mathrm{~m}$

$+, 1 \mathrm{y} 6 \mathrm{~m}$

†,3ylm

$+, 6 \mathrm{~m}$

$+, 7 \mathrm{~m}$

†, 1 y $2 \mathrm{~m}$

$+, 10 \mathrm{~m}$

$+, 11 \mathrm{~m}$

t,1y

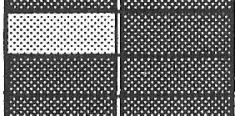

$\dagger, 10 \mathrm{~m}$

$+, 1 \mathrm{y}$

$+, 4 \mathrm{~m}$

$0,5 \mathrm{y} 1 \mathrm{~m}$

$\dagger, 9 \mathrm{~m}$

†, $1 \mathrm{y} 8 \mathrm{~m}$

$\dagger, 1 \mathrm{~m}$

t, $2 \mathrm{y}$

†, $1 \mathrm{y} 3 \mathrm{~m}$

$+, 1 \mathrm{y} 4 \mathrm{~m}$

$+, 2 \mathrm{y} 2 \mathrm{~m}$

t,1y $1 \mathrm{~m}$

+,1y6m

†, $1 \mathrm{y} 7 \mathrm{~m}$

$+, 3 y$

$+, 1 \mathrm{~m}$

$0,3 \mathrm{y} 9 \mathrm{~m}$

$+, 4 \mathrm{~m}$

†, 2 y $5 \mathrm{~m}$

$+, 1 \mathrm{y}$

$+, 0.3 \mathrm{~m}$

†,2y $5 \mathrm{~m}$

$+, 4 \mathrm{~m}$

$+, 3 \mathrm{~m}$

$+, 10 \mathrm{~m}$

\begin{tabular}{|l|l|}
\hline 1 & 0 \\
\hline 1 & 1 \\
\hline 1 & 1 \\
\hline 1 & 1 \\
\hline 1 & 1 \\
\hline 1 & 1 \\
\hline 1 & 1 \\
\hline 1 & 1 \\
\hline 1 & 1 \\
\hline 1 & 1 \\
\hline 1 & 1 \\
\hline 1 & 2 \\
\hline 1 & 2 \\
\hline 1 & 2 \\
\hline 2 & 0 \\
\hline 2 & 0 \\
\hline 2 & 1 \\
\hline 2 & 1 \\
\hline 2 & 1 \\
\hline 2 & 1 \\
\hline 2 & 1 \\
\hline 2 & 1 \\
\hline 2 & 1 \\
\hline 2 & 1 \\
\hline 2 & 1 \\
\hline 2 & 1 \\
\hline 3 & 0 \\
\hline 3 & 0 \\
\hline 3 & 0 \\
\hline 3 & 0 \\
\hline 3 & 0 \\
\hline 3 & 0 \\
\hline 3 & 1 \\
\hline 3 & 1 \\
\hline 3 & 1 \\
\hline 3 & 1 \\
\hline
\end{tabular}

$\mathrm{O}$ : alive

t: died

Fig. 1. Staining results in each patient, with follow-up data, lymphatic permeation status in and around the pancreas ('ly': 0: negative, 1: mild, 2: moderate, 3: marked), and lymph node metastasis status (' $n$ ': regional lymph nodes: $1:$ neighboring, $2:$ distaant). 
lymphatic status.

\section{Intrahepatic bile-duct tumor}

Secondly, we examined two kinds of intrahepatic bile-duct tumors (bile duct cyst adenocarcinomas with non-invasive character 9 , cholangiocarcinomas with marked invasive character 17) (9). Fig. 3 shows staining results in each patient, with follow-up data, and lymphatic permeation status and perineural invasion status in and around the tumors. There was a distinct difference between the survival of patients with bile duct cyst adenocarcinoma and that with cholangiocarcinoma. Tn and STn were rarely expressed or com-

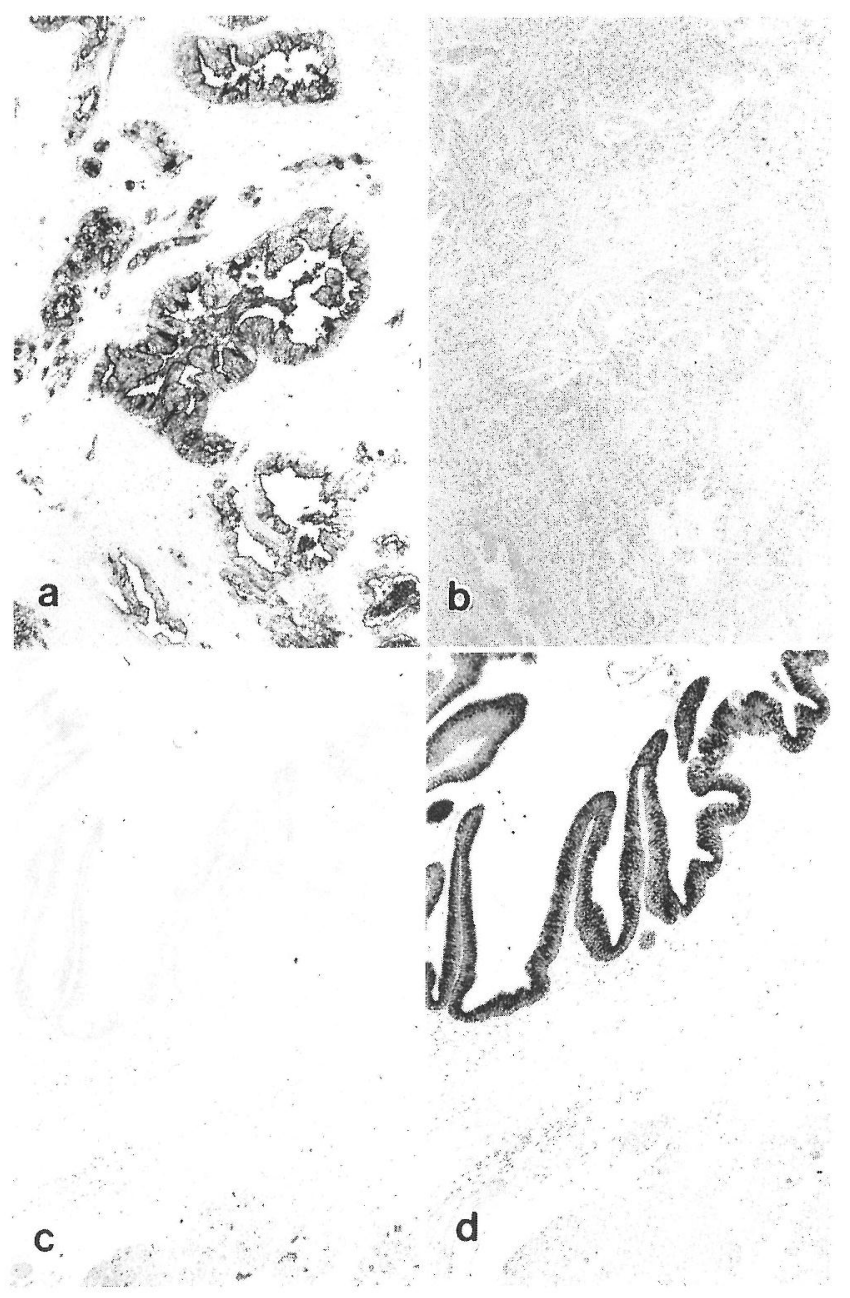

FIG. 2. Immunohistochemical stainings of MUC1 gene product (DF3) and MUC2 gene product (MRP) between invasive ductal carcinoma and intraductal papillary tumor. Invasive ductal carcinoma is DF3 positive (a) but MRP negative (b), whereas intraductal papillary tumor is DF3 negative (c) but MRP positive (d). $\quad \times 55$ pletely negative in the normal intrahepatic bile duct, whereas both antigens showed high expression rates in both bile duct cyst adenocarcinoma and cholangiocarcinoma. Non-invasive bile duct cyst adenocarcinomas with a good prognosis either expressed no MUC1 apomucin with $[\mathrm{DF} 3(-), \operatorname{MUSE} 11(-)$ and $139 \mathrm{H} 2(-)]$ pattern or expressed MUC1 apomucin with [DF3(-), MUSE11(+) and 139H2(+)] pattern. However these tumors often expressed MUC2 apomucin with $[$ anti-MRP(+) and CCP58(+)] pattern. In contrast, most invasive cholangiocarcinomas with a poor prognosis expressed MUC1 apomucin, but expressed no MUC2 apomucin.

\section{Ovary}

Mucinous ovarian tumors (benign 34, borderline 16, malignant 20) and serous ovarian tumors (benign 33 , borderline 7 , malignant 13) were examined (8).

Neither Tn nor STn antigen was expressed in non-neoplastic ovarian tissues. In contrast, the simultaneous expression of $\mathrm{Tn}$ and STn antigens was seen in all the mucinous and serous carcinomas. In the borderline tumors, this simultaneous expression of both antigens was observed in $69 \%$ of the mucinous tumors and in $57 \%$ of the serous tumors, whereas the simultaneous expression of Tn and STn antigens was not seen in the adenomas, except for a few mucinous adenomas.

DF3 expression rates in ovarian serous tumors were $70 \%$ in the adenoma, $100 \%$ in the borderline malignancy, and $100 \%$ in the carcinoma. MRP was completely negative in all the serous tumors of benign, borderline malignancy, and malignant. On the other hand, ovarian mucinous adenocarcinomas showed $\mathrm{DF} 3(+)$ and $\mathrm{MRP}(-)$ pattern in $45 \%$ of the cases, and DF3(+) and MRP $(+)$ pattern in $55 \%$. The MRP expression rate increased in accordance with the progression of the mucinous tumors from a benign to malignant state (adenoma 15\%, borderline malignancy $44 \%$, carcinoma 55\%). Co-expression of DF3 and MRP was seen in borderline malignancies (31\%) and carcinomas (55\%) of the mucinous tumors, although it was not seen in mucinous adenomas.

\section{Breast}

Finally, invasive ductal carcinomas of the breast (scirrhous type 25, non-scirrhous type 30) were examined (5). In the normal ducts, only DF3 was positive, whereas the other $\mathrm{Tn}, \mathrm{STn}$ and MRP were not found in the normal breast tissue. In the breast cancers, DF3 was expressed in 95\% but MRP was not expressed. Tn showed relatively high expression rate $(66 \%)$, whereas STn showed low expression rate 
(14\%). Simultaneous expression of $\mathrm{Tn}$ and $\mathrm{STn}$ was observed in $10 \%$ of the breast cancers. In scirrhous carcinomas, the Tn expression was seen in $60 \%$ of the cases with positive lymph node metastasis, whereas that was seen in $10 \%$ of the cases with negative lymph node metastasis $(\mathrm{p}<0.05)$.

\section{DISCUSSION}

In adenocarcinomas of the pancreas, intrahepatic bile-duct, and ovary, the core region epitopes of mucin oligosaccharides, Tn and STn were highly effective tumor markers, because of the high expression rates in cancer tissues but the rare expression in normal tissues. The current study also disclosed frequent simultaneous expression of $\mathrm{Tn}$ and $\mathrm{STn}$ in cancers of those organs, indicating that incomplete and aberrant glycosylation occurred in the course of cancerization. On the other hand, low expression rate of STn in breast cancers suggests that aberrant glycosylation pathways resulting in formation of $\mathrm{STn}$ is not so frequent in breast cancers. It was an interesting finding that a close association of Tn expression with lymph node metastasis was observed in the scirrhous type of breast cancer.

As for the expression of apomucins in the pancreatic and intrahepatic bile-duct tumors, a pattern of MUC1(+) and MUC2(-) was found in the invasive

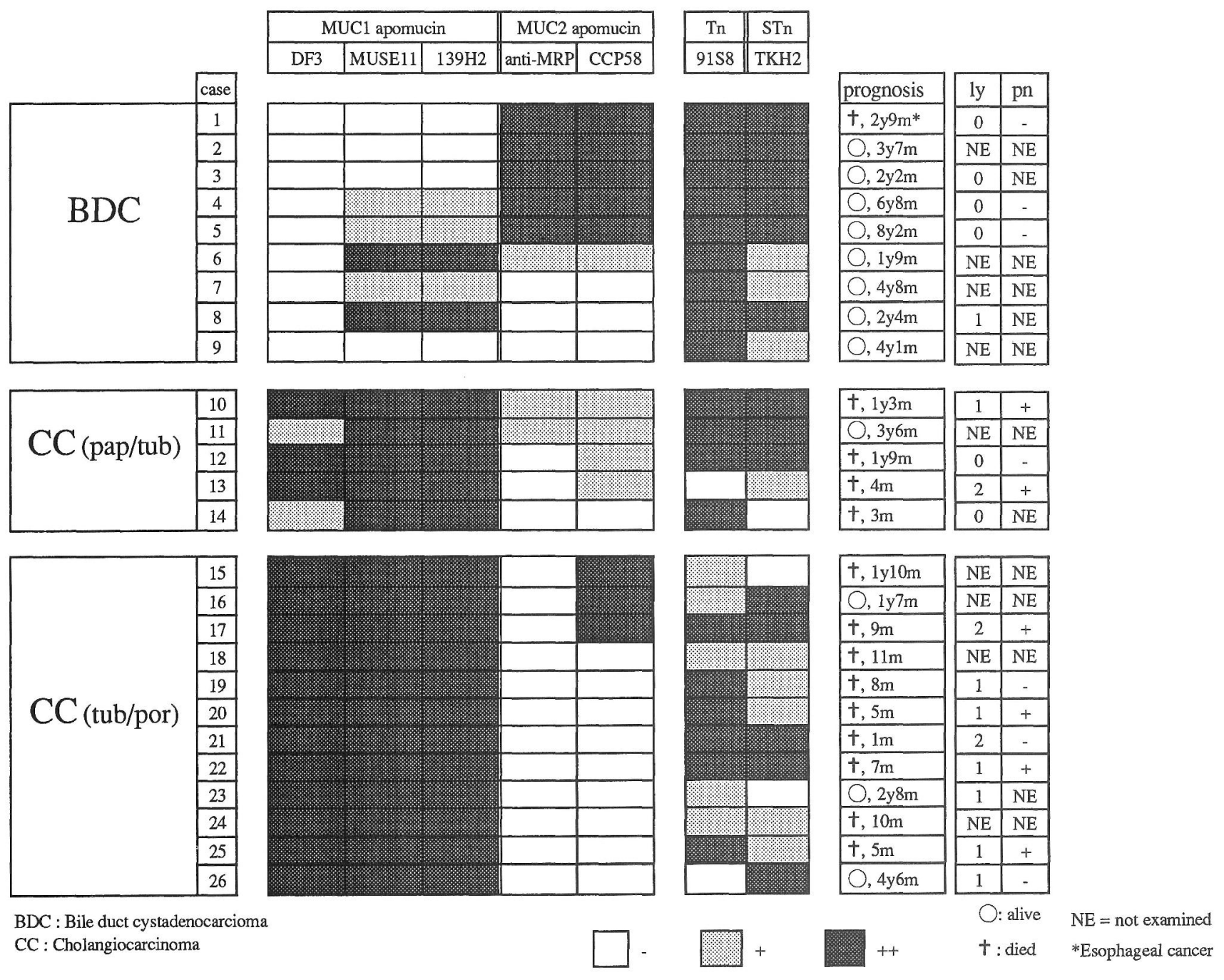

Fig. 3. Staining results in each patient, with follow-up data, and lymphatic permeation status ('ly': 0: negative, 1: mild, 2: moderate) and perineural invasion status ('pn') in and around the tumors. 
carcinomas with a poor prognosis, whereas MUC1( -$)$ and MUC2(+) pattern was frequently seen in non-invasive carcinomas with a favorable prognosis. Two possible explanations for this phenomenon may be possible: (1) apomucins produced by invasive carcinomas and non-invasive carcinomas are different; or (2) carcbohydrate chains block binding of the antibodies to the apomucins. Further in situ hybridization studies of MUC1 mRNA and MUC2 mRNA are necessary to obtain conclusive data regarding apomucin expression in those carcinomas with different growth pattern and prognosis.

The significance of MUC2 apomucin expression in the ovarian mucinous tumors seems to be different from that in the tumors of pancreas and intrahepatic bile-duct, because MUC2 apomucin was increasingly expressed in adenocarcinomas than in adenomas and in borderline tumors. The increased MUC2 apomucin expression and coexpression of MUC1 and MUC2 apomucins are indicative of the malignant potential of ovarian mucinous tumors.

\section{ACKNOWLEDGMENT}

This study was supported in part by Grant-in-Aid 06670196 from the Ministry of Education, Science and Culture, Japan.

\section{REFERENCES}

1. Gum, J. R.: Mucin genes and the proteins they encode: Structure, diversity, and regulation. Am. J. Respir. Cell. Mol. Biol. 7; 557-564, 1992.

2. Itzkowitz, S. H., Yuan, M., Montgomery, C. K., Kjeldsen, T., Takahashi, H.K., Bigbee, W. L. and Kim, Y.S.: Expression of Tn, sialosyl-Tn, and T antigens in human colon cancer. Cancer Res. 49; 197-204, 1989.
3. Kim, Y.S. and Byrd, J. C.: Colonic and pancreatic mucin glycoproteins expressed in neoplasia. In "Biochemical and Molecular Aspect of Selected Cancers”, vol. 1, ed. by T. G. II Pretlow and T. P. Pretlow. Academic press, 1991, pp. 277-311.

4. Kjeldsen, T., Clausen, H., Hirohashi, S., Ogawa, T., Iilima, H. and Hakomori, S.: Preparation and characterization of monoclonal antibodies directed to the tumorassociated O-linked sialosyl-2-6 $\alpha-\mathrm{N}$-acetylgalactosaminyl (sialosyl-Tn) epitope. Cancer Res. 48; 2214-2220, 1988.

5. Nomoto, M., Yonezawa, S., Tokunaga, M., Kim, Y. S. and Sato, E.: Mucin antigen expression and Ki-67 labeling in breast cancer: The peculiarity in scirrhous carcinoma. Pathol. Int., in press.

6. Osako, M., Yonezawa, S., Siddiki, B., Huang, J., Ho, J. J. L., Kim, Y. S. and Sato, E.: Immunohistochemical study of mucin carbohydrates and core protein in human pancreatic tumors. Cancer 71; 2191-2199, 1993.

7. Takahashi, H. K., Metoki, R. and Hakomori, S.: Immunoglobulin G3 monoclonal antibody directed to Tn antigen (tumor-associated $\alpha$-N-acetylgalactosaminyl epitope) that does not cross-react with blood group A antigen. Cancer Res. 48; 4361-4367, 1988.

8. Tashiro, Y., Yonezawa, S., Kim, Y. S. and Sato, E.: Immunohistochemical study of mucin carbohydrates and core protein in human ovarian tumors. Hum. Pathol. 25; 364-372, 1994.

9. Yamashita, K., Yonezawa, S., Tanaka, S., Shirahama, H., Sakoda, K., Imai, K., Xing, P-X., McKenzie, I. F. C., Hilkens, J., Kim, Y.S. and Sato, E.: Immunohistochemical study of mucin carbohydrates and core protein in hepatolithiasis and cholangiocarcinoma. Int. $J$. Cancer. 55; 82-91, 1993.

10. Yonezawa, S., Byrd, J. C., Dahiya, R., Ho, J. J. L., Gum, J. R., Griffiths, B., Swallow, D. M. and Kim, Y.S.: Differential mucin gene expression in human pancreatic and colon cancer cells. Biochem. J. 276; 599-605, 1991.

11. Yonezawa, S., Tachikawa, T., Shin, S. and Sato, E.: Sialosyl-Tn antigen: Its distribution in normal human tissues and expression in adenocarcinomas. Am. J. Clin. Pathol. 98; 167-174, 1992. 